\title{
Inter-Parietal White Matter Development Predicts Numerical Performance in Young Children
}

\author{
Jessica F. Cantlon ${ }^{1}$, Simon W. Davis ${ }^{2}$, Melissa E. Libertus ${ }^{2}$, Jill Kahane ${ }^{2}$, Elizabeth M. \\ Brannon' ${ }^{2}$, and Kevin A. Pelphrey ${ }^{3}$ \\ ${ }^{1}$ Department of Brain \& Cognitive Sciences, University of Rochester \\ ${ }^{2}$ Department of Psychology \& Neuroscience and Center for Cognitive Neuroscience, Duke \\ University \\ ${ }^{3}$ Yale Child Study Center, Yale University
}

\begin{abstract}
In an effort to understand the role of interhemispheric transfer in numerical development, we investigated the relationship between children's developing knowledge of numbers and the integrity of their white matter connections between the cerebral hemispheres (the corpus callosum). We used diffusion tensor imaging (DTI) tractography analyses to test the link between the development of the corpus callosum and performance on symbolic and non-symbolic numerical judgment tasks. We were especially interested in the interhemispheric connections of parietal cortex in 6-year-old children, because regions of parietal cortex have been implicated in the development of numerical skills by several prior studies. Our results revealed significant structural differences between children and adults in the fibers of the corpus callosum connecting the left and right parietal lobes. Importantly, these structural differences were predictive of individual differences among children in performance on numerical judgment tasks: children with poor numerical performance relative to their peers exhibited reduced white matter coherence in the fibers passing through the isthmus of the corpus callosum, which connects the parietal hemispheres.
\end{abstract}

\section{Introduction}

The corpus callosum is the largest white matter structure in the brain and functions to coordinate information between the cerebral hemispheres (see Gazzaniga, 2000 for review). It is a critical structure for sensory and motor integration (e.g., Eliassen et al., 1999) and it is also an important component of the neural networks recruited during higher-order cognitive processes including comparison (e.g., Brown et al., 1999), executive function (Bookstein et al., 2002), attention (Dorion et al., 2002), and language (Dougherty, et al., 2007; McDonald et al., 2001). Here, we examine the role of the corpus callosum in predicting performance on numerical judgment tasks. In particular, we ask whether the development of interhemispheric transfer in parietal cortex (via the fibers traversing the isthmus of the corpus callosum) is related to mastery of numerical skills in children, since parietal cortex is known to play a critical role in adult numerical cognition (see Dehaene et al., 2003 for review).

\footnotetext{
(C) 2011 Elsevier Inc. All rights reserved.

Publisher's Disclaimer: This is a PDF file of an unedited manuscript that has been accepted for publication. As a service to our customers we are providing this early version of the manuscript. The manuscript will undergo copyediting, typesetting, and review of the resulting proof before it is published in its final citable form. Please note that during the production process errors may be discovered which could affect the content, and all legal disclaimers that apply to the journal pertain.
} 
Abnormalities in the structure of the corpus callosum have been linked to a broad set of developmental disorders. Morphological abnormalities in the shape and size of the corpus callosum have been found in children with autism, schizophrenia, ADHD, and dyslexia (e.g., Egaas et al., 1995; Hynd et al., 1995; see Bloom \& Hynd, 2005 for review). Recent evidence has further established the link between the structural development of the corpus callosum and the development of cognitive functioning. For example, 18- to 40-month-old children with mental retardation due to generalized developmental delay exhibit reduced white matter volume in the corpus callosum relative to typically developing children (Cascio et al., 2006). A recent study with typically developing older children has also identified a link between the corpus callosum and general intelligence: children with higher IQ have significantly thicker callosal tracts than children with low IQ, independently of overall brain size (Luders et al., 2007). These data suggest that the corpus callosum may be a key substrate driving individual differences in cognitive development. Another recent study has found that the size of the corpus callosum correlates with lateralization of language development in children (Josse et al., 2008), indicating that asymmetries in brain function vary over development and correlate with corpus callosum development.

Coordination between hemispheres is likely an important feature of numerical processing. Brain imaging studies of adults engaged in numerical comparison tasks typically show bilateral intraparietal sulcus (IPS) activation (see Dehaene et al., 2003). Bilateral IPS activation is not critical, however, because neuropsychological studies of corpus callosotomy patients demonstrate that adults are able to make numerical comparisons of Arabic numerals, number words, and dot arrays within either hemisphere (Colvin, Funnell, \& Gazzaniga, 2005). And, although basic numerical computations in adulthood appear to recruit both hemispheres in fMRI studies, studies of numerical cognition in children suggest that hemispheric asymmetries in parietal cortex may be quite pronounced early in development (Ansari et al., 2005; Ansari \& Dhital, 2006; Cantlon et al., 2006; Rivera et al., 2005). For example, 4-year-old children exhibit more strongly right lateralized BOLD activity in the IPS than adults during non-symbolic numerical comparisons of arrays of dots (Cantlon et al., 2006). Older children (7- to 9-year-olds) also display right-lateralized IPS activity when comparing small Arabic numerals (i.e., digits 1 to 9; Holloway \& Ansari, in press). However, when comparing large Arabic numerals that are not highly familiar, the same-age children exhibit greater activity in the left parietal hemisphere (Cantlon et al., 2009) and a similar left-hemisphere bias has been reported for adult corpus callosotomy patients who are asked to complete difficult numerical computations with large numbers (Funnell, Colvin, \& Gazzaniga, 2007).

Children are equipped with the ability to represent number nonverbally from an early age however, their ability to understand, compare and manipulate numerical symbols such as written number words and digits is gradually acquired between 4 years of age and mid- to late-childhood. For example, adults perform symbolic numerical comparisons more accurately than and almost twice as quickly as 6- to 7-year-old children (e.g., Girelli, Lucangeli, \& Butterworth, 2000; Temple \& Posner, 1998). Additionally, for children, digits do not automatically call up their meanings. Unlike adults, children only begin to show Stroop interference and automatic representation of Arabic numerals and number words at 8 or 9 years of age (e.g., Girelli et al., 2000). Thus, there are a number of important cognitive changes that occur between early childhood and adulthood in numerical processing. The functional and structural changes that support numerical development at the neural level are less clear.

Structural analyses of human white matter development in vivo have revealed that development of white matter typically increases steadily throughout the brain between 4 and 20 years of age following a trajectory from a rostral-lateral-ventral pole to a dorsal-medial- 
caudal pole (Giedd et al., 1996; Giedd et al., 1999; see Toga et al., 2006 for review). These changes can occur in a number of different microstructural features: the number and size of axons, the common orientation of axons, brain water content, membrane density, glial population, and the myelination of axons (see Neil et al., 2002 for review). All of these features are important for the integrity of information transmission throughout the brain.

Diffusion tensor imaging (DTI) measures both the direction and degree of water diffusion along an axis (see LeBihan et al., 2001 for review). Due to the coherent organization of neural fibers in the fatty white matter, water diffuses in a highly directional or, anisotropic, manner along the axis of a white matter tract. Consequently, brain regions that reflect high degrees of anisotropic water diffusion signify the location of a robust white matter tract. Fractional anisotropy (FA) is a scalar quantity that represents the degree to which water diffuses in a common direction at a particular location (see Mori \& Zhang, 2006). On the basis of the FA index, several recent studies with children have demonstrated that white matter coherence in specific brain regions can yield important predictions about cognitive and brain functioning (Beaulieu, et al., 2005; Dougherty, et al., 2007; Nagy et al., 2004; Olesen, Nagy, Westerberg, \& Klingberg, 2003; Schmithorst, et al., 2005).

However, in contrast to the more common scalar, volumetric techniques, fiber-based tractography analyses use information from the tensor field to infer both the degree of anisotropic water diffusion (e.g., FA) and the specific direction of diffusion. Tractography analyses compute diffusion trajectories through a 3-dimensional image following the path with the greatest common diffusion (i.e., longest eigenvector) between pre-defined source and target regions (Mori \& van Zijl, 2002). Since this method provides information about both the intensity and direction of diffusion, it presents an opportunity to track specific white matter pathways through the brain.

In the current study, we used fiber-based tractography to examine the integrity of the corpus callosum in adults and young children (6- to 7-year-olds) as a correlate of behavioral performance on a basic cognitive task: numerical comparison. We used correlation analyses to test the relationship between measures of corpus callosum development and performance on the numerical tasks.

We examined the white matter integrity of the corpus callosum in three regions of interest: the genu (prefrontal), isthmus (superior parietal), and splenium (occipitotemporal). The superior parietal fibers were selected to test our hypotheses that inter-parietal connectivity influences numerical performance. Callosal tracts of the prefrontal hemispheres were selected because frontal regions also exhibit age-related changes in the degree to which they are engaged during numerical tasks (e.g., Ansari et al., 2005; Cantlon et al., 2009; Rivera et al., 2005). The fibers traversing the splenium were selected as a control tract.

\section{Methods}

\section{Subjects}

Eighteen children ( $6.89 \pm .62$ years, 9 male) and 14 adults ( $23.2 \pm 3.06$ years, 9 male) with normal or corrected vision participated in the experiment. None of our participants had any history of psychiatric disorder. The study met criteria for approval from the Duke University Institutional Review Board. Written informed consent was obtained from each adult participant and from the parent or guardian of each child. Children and adults were recruited from the Durham, North Carolina community. 


\section{Task}

All participants completed the same numerical judgment task inside the MRI scanner, prior to the DTI scan (Figure 1). On each trial, participants were required to identify the larger of two numerical values on a computer screen. Participants responded by pressing a joystick button corresponding to the side of the selected numerical value (left or right). The response window in which subjects could make their choice was two seconds. An image of a sun followed correct responses while a black screen followed incorrect responses and failures to respond within the two-second response window. Each run lasted six minutes (72 trials), and participants completed four runs. Two of the runs required participants to judge Arabic numerals (Symbolic condition) and the remaining two runs tested numerical judgments of dot arrays (Non-symbolic condition). The numerical values were identical in the two conditions and the values ranged from 2 to 56 . The specific numerical pairs tested were identical in the symbolic and non-symbolic conditions and included all pairs that formed either a 0.5 ratio or a 0.8 ratio when dividing the smaller by the larger value (rounded to the nearest tenth). Participants' accuracy and reaction time were recorded for each trial. During data analysis, subjects' accuracies were averaged for each ratio and then those accuracies were averaged to create an overall average of accuracy for the symbolic and non-symbolic conditions for each subject.

\section{DTI Acquisition \& Preprocessing}

Scanning was completed on a GE Signa EXCITE HD with 40-mT/m gradients at $150 \mathrm{~T} / \mathrm{m} / \mathrm{s}$ slew rate at the Duke University Medical Center. The DT-MRI dataset was based on a single-shot EPI sequence $(\mathrm{TE}=86 \mathrm{~ms}, \mathrm{TR}=17000 \mathrm{~ms}, 50$ contiguous slices of $2.0 \mathrm{~mm}$ thickness, FoV $=256 \times 256 \mathrm{~mm}^{2}$, matrix size $128 \times 128$, voxel size $2 \times 2 \times 2 \mathrm{~mm}, 1$ nondiffusion weighted scan $\left(b\right.$-value $\left.=1000 \mathrm{~s} / \mathrm{mm}^{2}\right), 15$ diffusion-sensitizing directions, 960 total images, total scan time approx.. 5 minutes). Non-brain and ambient noise were removed from the diffusion weighted images using the Brain Extraction Tool (BET; Smith, 2002). An internally developed Matlab script condensed the 960 Dicom images into sixteen diffusion weighted images (DWIs). These DWIs were eddy-corrected and used to determine diffusion tensors in the computer program DTI Track 2005 (Pierre Fillard). Tensor construction was based on the fit of the tensor model to the diffusion data. Three eigenvectors, or diffusivities, $\left(\lambda_{1}>\lambda_{2}>\lambda_{3}\right)$ were generated by diagonalization of the tensor. These voxel-specific eigenvectors represent the diffusivities throughout the three directions of the tensor and were the foundation of the diffusion properties investigated in our study.

\section{Identification of Seed and Target Regions}

Seed and target brain regions were traced on subjects' anatomical images in order to narrow the scope of the fiber tracking algorithms to specific regions of interest (ROIs). The computer program ITK/SNAP (Guido Gerig, 2004) was used to trace the corpus callosum (seed region) and the target ROIs for each subject. We defined our seed region as the midsagittal plane of the corpus callosum (white matter) and the target tracts were defined as those crossing the two sagittal slices adjacent to the midsagittal plane into cortex. Thus, to be included in our analysis, a tract had to cross the midsagittal plane of the corpus callosum and radiate out of the corpus callosum into cortex. We identified the subsections of the corpus callosum using an atlas. The Witelson method (1989) was used for the subdivision of the corpus callosum. The target region for the splenium-parietal fibers was the isthmus of the corpus callosum, and was located on 3-5 sagittal slices, using the presence of the body of the corpus callosum in the axial view as a superior boundary, and the top of the lateral ventricle as an inferior boundary. Anatomical borders for source regions were two parasagittal planes, $6-8$ sagittal slices from the axial midline, including the parietal lobe posterior to the postcentral gyrus and anterior to the occipital-parietal sulcus. To isolate fibers connecting the splenium and parietal lobe, tract estimation was constrained to exclude 
any fibers terminating outside these borders. The central sulcus was used as a reference point for the placement of segmentation regions, and a diffusion atlas (Scarabino et al., 2004) provided further anatomical guidelines for locating the tracts of interest and corresponding gray matter target ROIs.

\section{Fiber Tracking}

The construction of fiber tracts was completed using FiberTracking software (UNC Chapel Hill). A fractional anisotropy (FA) map was computed for each subject using his or her tensor data and the traced seed and target regions. The three-dimensional fiber tracts were then constructed for each ROI using an edge-step algorithm that generated each tract on a voxel-by-voxel basis by continuously following the direction of greatest diffusivity in each voxel between the seed and the target ROIs (see above). An FA threshold was set at 0.2, and a curvature threshold (angle between the first eigenvector and the eigenvector in the next voxel) was set to $40^{\circ}$. The three fiber tracts (traversing the genu, splenium, and isthmus) were then evaluated according to a priori knowledge of adult tract organization (Wakana, Jiang, Nagae-Poetscher, van Zijl, \& Mori, 2004), and edited in FiberViewer 1.2.3 (Sylvain Gouttard \& Matthieu Jomier, 2004) to remove outlier fibers that are a common artifact of the tracking algorithms used in tractography. Grouping algorithms were applied to refine tracts into fiber bundles that maintained directional coherence and common length. Thresholds cutting off the lower and upper bounds of fiber length were determined using a histogram of fiber lengths for each tract. The lower and upper threshold values were set at 50 and 150 , respectively. This analysis technique yielded fiber tracts representing callosal pathways through the genu, isthmus, and splenium that closely paralleled the representations of Scarabino et al., (2004) and a validated DTI tractography atlas (Wakana, et al., 2007). The average number of fibers in the entire cortex was 920 for adults and 715 for children (adult $\mathrm{SD}=250$ fibers, child $\mathrm{SD}=400$ fibers). The average number of fibers crossing the isthmus was similar for both children and adults (adults: mean $=243, \mathrm{SD}=106$, child: mean $=232 \mathrm{SD}=85$ ). Mean fiber length in the isthmus tract was about 90 millimeters for both adults and children.

Diffusion properties, including FA, axial $\left(\lambda_{1}\right)$ and radial diffusivity $\left(\left(\lambda_{2}+\lambda_{3}\right) / 2\right)$ were computed and evaluated at points along the cross section of each bundle of fiber tracts. Axial diffusivity occurs parallel to the primary direction of diffusivity, while radial diffusivity occurs perpendicular to the maximum (or, primary) diffusivity direction.

\section{DTI and Behavioral Analyses}

Structural DTI analyses measured FA, axial diffusivity, and radial diffusivity for the three ROIs (prefrontal/genu, parietal/isthmus, and occipitotemporal/splenium) in each participant. These analyses were conducted over the middle $50 \mathrm{~mm}$ of each tract as well as for smaller $5 \mathrm{~mm}$ subsections of each tract, including the midpoint, as described in the Results. Each tract was aligned at the mid-sagittal plane across subjects for group comparisons. Statistical contrasts were computed to quantitatively compare these structural measures between adults and 6-year-old children. These analyses are described in Results. Behavioral analyses were completed using regression analyses relating the diffusivity measurements in each ROI to the behavioral data for each participant. Reaction time and accuracy, both overall and separated by numerical notation (Arabic numerals or Dot Arrays), were the behavioral measures of interest.

\section{Results}

Our analyses revealed structural differences between children and adults in the interhemispheric white matter tracts connecting parietal and occipito-temporal cortices (i.e., 
through the isthmus and splenium). In addition, we found a relationship between behavioral performance on the numerical judgment tasks and white matter coherence in the superior parietal tract of the corpus callosum (isthmus) but not in the prefrontal (genu) or occipitotemporal tracts (posterior splenium). We describe these results in detail below.

\section{Age-related Structural Differences}

We compared the FA values along occipito-temporal (splenium), superior parietal (isthmus), and prefrontal (genu) callosal tracts to determine whether adults exhibited significantly greater white matter content than children in these regions. Figure 2 shows the locus of each tract in a sample 6-year-old child. A repeated measures ANOVA comparing Group (Adults, Children) $\times$ Tract $($ Genu, Isthmus, Splenium $) \times$ Hemisphere (Left, Right) revealed a main effect of Group $(F(1,90)=47.9, p<.001)$, a main effect of Tract $(F(2,90)=19.8, p<.001)$, and a marginally significant main effect of Hemisphere $(F(1,90)=3.36, p=.07)$. None of the interactions were significant. The majority of the differences between children and adults were in tracts traversing the isthmus and splenium and there were greater differences in the left hemisphere than the right (Figure 3). To validate this observation, we compared FA values at a regional level for each tract, averaging across lengths of five millimeters (FDR corrected $\alpha=.05$ ). Children exhibited significantly lower FA than adults at a small $5 \mathrm{~mm}$ length of the fibers traversing the left lateral genu and much larger extents $(10-15 \mathrm{~mm})$ of the left, right, and mid isthmus and splenium. Significant differences between children and adults along those tracts are highlighted in Figure 3. These results are consistent with prior studies showing that corpus callosum growth continues throughout the tract during early and late childhood but exhibits the greatest growth in more posterior sections (Rajapakse et al., 1996). One possible explanation of the more prolonged and dramatic growth in the more posterior callosal fibers is that these axons ultimately become more myelinated than those in anterior regions (Aboitiz, Lopez, \& Montiel, 2003).

As described earlier, the FA value is a summary statistic of diffusivity in all three spatial dimensions. Therefore, increases in the FA value could represent more coherent diffusivity along the primary axis or, alternatively, it could represent reduced diffusivity along perpendicular axes. To explore the source of the differences in FA between children and adults, we examined the diffusivity components of the FA value: axial diffusivity (AD) and radial diffusivity (RD). An ANOVA comparing Group $\times$ Tract on the dependent measure of $\mathrm{AD}$ revealed a main effect of Group $(F(1,90)=5.99, p<.01)$ and a main effect of Tract $(F(2,90)=26.22, p<.001)$ with no interaction $(p=0.99)$. The effect of Group is due to higher $\mathrm{AD}$ in children compared to adults ( $1.43 \mathrm{vs.} 1.39$, respectively) and the effect of Tract is due to greater $\mathrm{AD}$ in the genu compared to the other two tracts (both p's $<.001$ ). The same ANOVA was tested with RD as the dependent measure and it yielded the same main effects of Group and Tract with no interaction $(F(1,90)=38.3), p<.001 ; F(2,90)=18.9, p$ $<.001 ; p=0.46)$. These effects reflect the higher RD values of children compared to adults (5.9 vs. 5.3, respectively) and significant, step-like decreases in the magnitude of RD from the fibers traversing the genu to the isthmus to the splenium (all p's $<.02$ ).

Figures 4 and 5 show the differences between children and adults in $\mathrm{AD}$ and $\mathrm{RD}$ along each tract, measured in $5 \mathrm{~mm}$ increments (FDR corrected $\alpha=.05$ ). As can be seen in the figures, differences in RD between children and adults were more pronounced and extensive than differences in AD. However, the only whole tract that showed an overall difference between adults and children in $\mathrm{RD}$ versus $\mathrm{AD}$ was the splenium, in which $\mathrm{RD}$ was significantly more different between adults and children than $\mathrm{AD}(\mathrm{t}(60)=8.3, p<.001)$. $\mathrm{AD}$ is a white matter metric more attuned to the number and intrinsic characteristics of axons or changes in extraaxonal/extracellular space. RD, on the other hand, has been associated with changes in myelination or glial cell morphology (Song, et al., 2003; Song, et al., 2005). Therefore, one possibility is that children exhibit more extensive differences from adults in RD than $\mathrm{AD}$ 
due to immature myelination. In particular, the strong dissociation between RD and AD in the more posterior segments of the corpus callosum fits with the argument, described earlier, that more posterior fibers ultimately become more myelinated than anterior fibers.

\section{Brain-Behavior Correlations}

Since there were clear differences in white matter coherence between children and adults, our next question was whether these structural measures were related to children's behavioral performance. FA values for each tract were tested against participants' performance on our two numerical judgment tasks: Symbolic (Arabic numerals) and Nonsymbolic (arrays of dots).

The behavioral results from these two tasks are shown in Figure 6. An ANOVA testing Age Group (Adults, Children) $\times$ Condition (Symbolic, Non-symbolic) on accuracy revealed main effects of Age Group $(F(1,30)=23.9, p<.001)$ and Condition $(F(1,30)=21.0, p<.001)$ with no interaction. Adults were more accurate than children $(93 \%$ vs $79 \%, p<.001)$ and both groups performed better with Arabic numerals than Dot Arrays (88\% vs. 82\%, $p<$. 001). A parallel ANOVA on RTs revealed a significant interaction between Age Group and Condition $(F(1,30)=53.8, p<.001)$ wherein adults had faster RTs to Arabic numerals than Dot arrays but children showed no difference in RT between the two conditions (Figure 6b). Adults were also significantly faster than children overall ( 738 vs. $1056 \mathrm{msec}$, respectively, $p<.001)$. Thus, there are both speed and accuracy differences between children and adults in numerical comparison. Our goal was to understand whether structural differences in the corpus callosum could explain some of the variability in performance between groups.

A multiple regression model with accuracy as the dependent measure and FA values for the left and right genu, isthmus, and splenium as predictors was significant as a whole $\left(\mathrm{R}^{2}=\right.$ 0.50 , Adj. $\left.\mathrm{R}^{2}=0.38, F(6,25)=4.1, p<.005\right)$ and the only significant Beta coefficient in the model was from the fibers crossing the left isthmus (Beta $=0.61, t(25)=2.74, p<.01)$. All other Beta coefficients were less than 0.2. Similarly, the coefficient for the fibers that crossed the left isthmus was also marginally significant when the categorical factor of Age Group was included in the model (Beta $=0.44, t(24)=1.85, p=.06$ ). This expanded model was significant as a whole $\left(\mathrm{R}^{2}=0.57\right.$, Adj. $\left.\mathrm{R}^{2}=0.45, F(7,24)=4.6, p<.005\right)$ and the main effect of Age Group was marginally significant $(F(1,24)=4.23, p=.05)$. However, the expanded model did not account for substantially more variance than the original model without the factor of Group (Mallow's $C_{p}=8$ vs. 7 for the models with and without the Age Group factor, respectively). Thus, the relationship between accuracy on numerical tasks and white matter coherence (FA) is not entirely explained by group differences.

A similar pattern emerged from regression analyses with RT as the dependent variable. A model with the left and right genu, isthmus, and splenium as predictors was significant as a whole $\left(\mathrm{R}^{2}=0.54\right.$, Adj. $\left.\mathrm{R}^{2}=0.43, F(6,25)=4.9, p<.005\right)$ and the only significant Beta coefficient in the model was from the tract that traversed the left isthmus (Beta $=-0.52$, $t(25)=-2.5, p<.05)$. When Age Group was entered as an additional predictor, the whole model was significant $\left(\mathrm{R}^{2}=0.69\right.$, Adj. $\left.\mathrm{R}^{2}=0.61, F(7,24)=7.9, p<.001\right)$ and the factor of Age Group was significant $(F(1,24)=12.5, p<.01)$. The highly significant factor of Age Group in predicting RT suggests that group differences might have played a greater role in the relationship between FA and RT than FA and accuracy.

To further investigate the relationship between FA and behavior, we conducted separate regression analyses for each numerical judgment type (Symbolic, Non-symbolic) and each age group. Figure 7 shows the raw correlation coefficients for the dependent variables of accuracy and RT and the FA values of the tract that crossed the left isthmus (the parietal corpus callosum). These analyses demonstrate that there is an overall relationship between 
the robustness of the left parietal corpus callosum and performance (Figure 7a \& b: Accuracy $\left.\mathrm{R}^{2}=0.41, p<.001 ; \mathrm{RT} \mathrm{R}^{2}=0.45, p<.001\right)$. That relationship persists across both the Symbolic and Non-symbolic numerical judgments (Figure 7c \& d): performance on both the Arabic numerals and Dot Arrays judgments were each significantly correlated with FA of the left parietal corpus callosum for both accuracy (Symbolic $\mathrm{R}^{2}=0.41, p<.001$; Nonsymbolic $\mathrm{R}^{2}=0.30, p<.01$ ) and $\mathrm{RT}$ (Symbolic $\mathrm{R}^{2}=0.47, p<.001$; Non-symbolic $\mathrm{R}^{2}=$ $0.30, p<.01$ ). When children's data were analyzed separately (Figure 7e \&f), FA in the left parietal tract was found to correlate with accuracy $\left(\mathrm{R}^{2}=0.24, p<.05\right)$ but the relationship did not hold for adults. Thus, group differences between children and adults account for a significant amount of variance in the correlation between performance and FA in the left parietal tract however, the relationship between FA and performance in the left parietal tract also explains within-group variability for 6-year-old children.

\section{Discussion}

Our results provide novel evidence of a link between the maturity of the corpus callosum and the development of numerical skills in 6-year-old children. Specifically, the development of the region of the corpus callosum connecting the superior parietal hemispheres (marked by the isthmus at the mid-sagittal plane) was related to performance differences between adults and children as well as to performance differences among children. These data provide the impetus for further exploration of the neuro-cognitive link between the development of the corpus callosum and numerical skill acquisition, particularly in parietal cortex.

We did not find a relation between the development of the region of the corpus callosum connecting the prefrontal hemispheres and numerical performance. It might be the case that interhemispheric connections between prefrontal regions are not as important as the connections between prefrontal cortex and other cortical regions such as the temporal and parietal cortices. In fact, recent studies with older children and adults have shown that the integrity of the superior and inferior longitudinal fasiculi correlates with symbolic calculation performance (Tsang et al., 2009; van Eimeren et al., 2008). Prefrontal connections could be more important for higher level calculation than basic numerical knowledge. The role of prefrontal cortex in numerical development is hypothesized to be a domain-general function such as semantic association learning, relational reasoning, or working memory (e.g., Cantlon et al., 2009; Tsang et al., 2009). In contrast, the role of parietal cortex in numerical development is hypothesized to be domain-specific (see Dehaene et al., 2003 for review).

White matter volume in parietal cortex has been linked to performance in children with mathematics-specific impairments due to Velocardiofacial syndrome. A prior voxel-based morphometry study tested white matter volume in individuals with numerical- and mathematics-related developmental disorders. In that study, 8- to 18-year-old children and adults with Velocardiofacial syndrome exhibited reduced white matter volume along the length of the intraparietal sulcus, the angular gyrus, and the supramarginal gyrus, contingent on the degree of their impairment in mathematics (Barnea-Goraly et al., 2005). Furthermore, a recent DTI study with 7- to 9-year-old children reported correlations between the left inferior longitudinal fasiculus (which connects occipital and temporal cortices) and the left superior corona radiata (which inputs to temporo-parietal cortex) with performance on a paper-and-pencil calculation test (van Eimeren et al., 2008; van Eimeren et al., 2010). Importantly, this correlation was specific to mathematics performance because measures of overall IQ were controlled in the model. Our results complement the conclusions of these two prior studies by providing evidence from typically developing, younger children 
indicating that the strength of the white matter connections between parietal hemispheres is linked to basic numerical performance.

Neuropsychological and functional neuroimaging studies in adults also implicate a specific role for intraparietal and superior parietal cortices in numerical judgments (see Dehaene et al., 2003 for review). In adults, bilateral regions of the intraparietal sulcus respond more strongly during numerical tasks than control tasks involving shape, color, letters, sentences, and a host of other stimuli. And, as described earlier, adults can compute numerical judgments within either hemisphere, as evidenced by studies of split-brain patients (Colvin, Funnell, \& Gazzaniga, 2005; Funnel, Colvin, \& Gazzaniga, 2007). Taken together, these studies provide evidence of bilateral, number-specific processing in regions of parietal cortex.

Developmental fMRI studies have shown that regions of parietal cortex become increasingly engaged by numerical tasks between the ages of 4 and 17 years, ultimately resulting in the adult pattern of bilateral parietal activation (e.g., Ansari et al., 2005; Ansari \& Dhital, 2006; Cantlon et al., 2006, 2009; Rivera et al., 2005). Early in development, children often show hemispheric asymmetries in parietal activation that are linked to immature numerical performance. For example, Rivera et al. (2005) reported that older children and adults engaged bilateral parietal and prefrontal regions during a basic arithmetic task, but younger children (who performed worse than older children) primarily engaged right hemispheric loci. Other studies testing numerical judgments have also reported right-lateralized parietal activity in young children (Holloway \& Ansari, in press; Cantlon et al., 2006). The hemispheric asymmetry in BOLD activity in children could be caused by the lack of mature white matter connections between hemispheres. The fact that children tend to exhibit rightlateralized parietal activation during numerical tasks whereas adults more often exhibit bilateral parietal activation might be related to the weak white matter composition of the left parietal tract in children compared to adults and in poor-performing children compared to other children (reported in the current study). Interhemispheric connections in parietal cortex may thus be an important catalyst for mature numerical representation. It is important to note, however, that our data do not rule out the possibility that the relationship between white matter coherence in the parietal corpus callosum and numerical performance is related to cognitive performance in general (rather than numerical performance in particular).

A current debate regarding the specific role of the corpus callosum in integrating the cerebral hemispheres is centered on the issue of the excitatory versus inhibitory influence of the corpus callosum on the contralateral hemisphere (Bloom \& Hynd, 2005). The basic question is whether the corpus callosum inhibits the contralateral hemisphere to generate hemispheric specialization and dominance or whether it excites the contralateral hemisphere to coordinate information in a joint information-processing effort. The answer to this question will be important for understanding developmental changes in the patterns of number-related activation between parietal hemispheres. At the moment, it is unclear whether the development of the left parietal corpus callosum is related to increased or decreased activation in the left parietal hemisphere $o r$, increased or decreased activation in the right parietal hemisphere. Combined fMRI and DTI studies of numerical development will help to resolve this issue.

Our data also prompt some conclusions about the structural development of the corpus callosum. The structural data demonstrate that differences between adults and children in FA and diffusivity indices varied among callosal tracts. This finding is consistent with prior reports that immature commissural pathways are not spatially homogeneous (Bermann, Mukherjee et al., 2005). In particular, we observed greater differences between children and adults in parietal and occipital callosal projections than frontal projections. Our observed 
differences in RD—greater than AD—in these tracts suggest that the age-related differences reported here are driven largely by myelin formation, consistent with other DTI studies of children in this age range (Lebel, Walker, Leemans, Phillips, \& Beaulieu, 2008; Paus, 2010). Myelination of the corpus callosum can thus occur at different rates for different cortical regions during early childhood.

In summary, our study provides evidence that structural differences in the white matter fibers of the corpus callosum that project between the parietal hemispheres are related to developmental progress in numerical performance in early childhood. Although prior fMRI studies implicate a specific role for intraparietal regions in numerical performance, the data from the current study do not afford any claims about the functional specificity of white matter development in superior parietal cortex. As mentioned earlier, studies of children with broad cognitive impairments have identified a link between the structure of the corpus callosum and general intelligence. Our results implicate a connection between white matter development and numerical performance that may either be specific to the numerical domain or, may be related to the development of general faculties such as attention and working memory. Parietal activation has previously been linked to those domain-general functions in adults and children (Casey, Galvan, \& Hare, 2005; Göbel et al., 2004;

Marshuetz et al., 2006), but domain-general activity also has been shown to dissociate from number-related activation in the intraparietal sulcus (e.g., Cantlon et al., 2009; Simon et al., 2002; see Dehaene et al., 2003 for review). Given the importance of parietal cortex for a wide range of cognitive functions, there is a strong possibility that the development of the parietal corpus callosum plays a specific role in the development of numerical cognition but also contributes to more general aspects of cognitive development.

\section{References}

Aboitiz F, Lopez J, Montiel J. Long distance communication in the human brain: timing constraints for inter-hemispheric synchrony and the origin of brain lateralization. Biol Res. 2003; 36(1):89-99. [PubMed: 12795208]

Ansari D. Paving the way towards meaningful interactions between neuroscience and education. Developmental Science. 2005; 8(6):466-467. [PubMed: 16246235]

Ansari D, Dhital B. Age-related changes in the activation of the intraparietal sulcus during nonsymbolic magnitude processing: An event-related functional magnetic resonance imaging study. Journal of Cognitive Neuroscience. 2006; 18(11):1820-1828. [PubMed: 17069473]

Ansari D, Garcia N, Lucas E, Hamon K, Dhital B. Neural correlates of symbolic number processing in children and adults. NeuroReport. 2005; 16(16):1769-1773. [PubMed: 16237324]

Barnea-Goraly N, Eliez S, Menon V, Bammer R, Reiss AL. Arithmetic ability and parietal alterations: A diffusion tensor imaging study in Velocardiofacial syndrome. Cognitive Brain Research. 2005; 25(3):735-740. [PubMed: 16260124]

Beaulieu C, Plewes C, Paulson LA, Roy D, Snook L, Concha L, et al. Imaging brain connectivity in children with diverse reading ability. NeuroImage. 2005; 25(4):1266-1271. [PubMed: 15850744]

Berman JI, Mukherjee P, Partridge SC, Miller SP, Ferriero DM, Barkovich AJ, et al. Quantitative diffusion tensor MRI fiber tractography of sensorimotor white matter development in premature infants. NeuroImage. 2005; 27(4):862-871. [PubMed: 15978841]

Bloom JS, Hynd GW. The role of the corpus callosum in interhemispheric transfer of information: Excitation or inhibition? Neuropsychology Review. 2005; 15(2):59-71. [PubMed: 16211466]

Bookstein FL, Streissguth AP, Sampson PD, Connor PD, Barr HM. Corpus callosum shape and neuropsychological deficits in adult males with heavy fetal alcohol exposure. NeuroImage. 2002; 15(1):233-251. [PubMed: 11771992]

Brown WS, Jeeves MA, Dietrich R, Burnison DS. Bilateral field advantage and evoked potential interhemispheric transmission in commissurotomy and callosal agenesis. Neuropsychologia. 1999; 37(10):1165-1180. [PubMed: 10509838] 
Cantlon JF, Brannon EM, Carter EJ, Pelphrey KA. Functional imaging of numerical processing in adults and 4-y-old children. Plos Biology. 2006; 4(5):844-854.

Cantlon JF, Libertus ME, Pinel P, Dehaene S, Brannon EM, Pelphrey KA. The neural development of an abstract concept of number. J Cogn Neurosci. 2009; 21(11):2217-2229. [PubMed: 19016605]

Cantlon JF, Platt ML, Brannon EM. Beyond the number domain. Trends in Cognitive Sciences. 2009; 13(2):83-91. [PubMed: 19131268]

Cascio CJ, Gerig G, Piven J. Diffusion tensor imaging: Application to the study of the developing brain. Journal of the American Academy of Child and Adolescent Psychiatry. 2007; 46(2):213223. [PubMed: 17242625]

Casey BJ, Galvan A, Hare TA. Changes in cerebral functional organization during cognitive development. Curr Opin Neurobiol. 2005; 15(2):239-244. [PubMed: 15831409]

Colvin MK, Funnell MG, Gazzaniga MS. Numerical processing in the two hemispheres: Studies of a split-brain patient. Brain and Cognition. 2005; 57(1):43-52. [PubMed: 15629214]

Dehaene S, Piazza M, Pinel P, Cohen L. Three parietal circuits for number processing. Cognitive Neuropsychology. 2003; 20(3-6):487-506. [PubMed: 20957581]

Dorion AA, Sarazin M, Hasboun D, Hahn-Barma V, Dubois B, Zouaoui A, et al. Relationship between attentional performance and corpus callosum morphometry in patients with Alzheimer's disease. Neuropsychologia. 2002; 40(7):946-956. [PubMed: 11900746]

Dougherty RF, Ben-Shachar M, Deutsch GK, Hernandez A, Fox GR, Wandell BA. Temporal-callosal pathway diffusivity predicts phonological skills in children. Proc Natl Acad Sci U S A. 2007; 104(20):8556-8561. [PubMed: 17483487]

Dubois J, Hertz-Pannier L, Cachia A, Mangin JF, Le Bihan D, Dehaene-Lambertz G. Structural asymmetries in the infant language and sensori-motor networks. Cereb Cortex. 2009; 19(2):414423. [PubMed: 18562332]

Egaas B, Courchesne E, Saitoh O. Reduced size of corpus callosum in autism. Arch Neurol. 1995; 52(8):794-801. [PubMed: 7639631]

Eliassen JC, Baynes K, Gazzaniga MS. Direction information coordinated via the posterior third of the corpus callosum during bimanual movements. Experimental Brain Research. 1999; 128(4):573577.

Funnell MG, Colvin MK, Gazzaniga MS. The calculating hemispheres: Studies of a split-brain patient. Neuropsychologia. 2007; 45(10):2378-2386. [PubMed: 17420034]

Gazzaniga MS. Cerebral specialization and interhemispheric communication - Does the corpus callosum enable the human condition? Brain. 2000; 123:1293-1326. [PubMed: 10869045]

Giedd JN, Blumenthal J, Jeffries NO, Castellanos FX, Liu H, Zijdenbos A, et al. Brain development during childhood and adolescence: a longitudinal MRI study. Nature Neuroscience. 1999; 2(10): 861-863.

Giedd JN, Snell JW, Lange N, Rajapakse JC, Casey BJ, Kozuch PL, et al. Quantitative magnetic resonance imaging of human brain development: Ages 4-18. Cerebral Cortex. 1996; 6(4):551560. [PubMed: 8670681]

Girelli L, Lucangeli D, Butterworth B. The development of automaticity in accessing number magnitude. Journal of Experimental Child Psychology. 2000; 76(2):104-122. [PubMed: 10788305]

Gobel SM, Johansen-Berg H, Behrens T, Rushworth MF. Response-selection-related parietal activation during number comparison. J Cogn Neurosci. 2004; 16(9):1536-1551. [PubMed: 15601517]

Holloway ID, Ansari D. Developmental Specialization in the Right Intraparietal Sulcus for the Abstract Representation of Numerical Magnitude. J Cogn Neurosci. 2009

Hynd GW, Hall J, Novey ES, Eliopulos D, Black K, Gonzalez JJ, et al. Dyslexia and Corpus-Callosum Morphology. Archives of Neurology. 1995; 52(1):32-38. [PubMed: 7826273]

Josse G, Seghier ML, Kherif F, Price C. Explaining function with anatomy: Language lateralization and corpus callosum size. Journal of Neuroscience. 2008; 28(52):14132-14139. [PubMed: 19109495]

Le Bihan D. Looking into the functional architecture of the brain with diffusion MRI. Nature Reviews Neuroscience. 2003; 4(6):469-480. 
Lebel C, Walker L, Leemans A, Phillips L, Beaulieu C. Microstructural maturation of the human brain from childhood to adulthood. NeuroImage. 2008; 40(3):1044-1055. [PubMed: 18295509]

Luders E, Narr KL, Bilder RM, Thompson PM, Szeszko PR, Hamilton L, et al. Positive correlations between corpus callosum thickness and intelligence. NeuroImage. 2007; 37(4):1457-1464. [PubMed: 17689267]

Marshuetz C, Reuter-Lorenz PA, Smith EE, Jonides J, Noll DC. Working memory for order and the parietal cortex: an event-related functional magnetic resonance imaging study. Neuroscience. 2006; 139(1):311-316. [PubMed: 16417974]

McDonald CR, Crosson B, Valenstein E, Bowers D. Verbal encoding deficits in a patient with a left retrosplenial lesion. Neurocase. 2001; 7(5):407-417. [PubMed: 11744782]

Mori S, van Zijl PC. Fiber tracking: principles and strategies - a technical review. NMR Biomed. 2002; 15(7-8):468-480. [PubMed: 12489096]

Nagy Z, Westerberg H, Klingberg T. Maturation of white matter is associated with the development of cognitive functions during childhood. Journal of Cognitive Neuroscience. 2004; 16(7):1227-1233. [PubMed: 15453975]

Neil J, Miller J, Mukherjee P, Huppi PS. Diffusion tensor imaging of normal and injured developing human brain - a technical review. Nmr in Biomedicine. 2002; 15(7-8):543-552. [PubMed: 12489100]

Olesen PJ, Nagy Z, Westerberg H, Klingberg T. Combined analysis of DTI and fMRI data reveals a joint maturation of white and grey matter in a fronto-parietal network. Brain Res Cogn Brain Res. 2003; 18(1):48-57. [PubMed: 14659496]

Paus T. Growth of white matter in the adolescent brain: myelin or axon? Brain Cogn. 2010; 72(1):2635. [PubMed: 19595493]

Rajapakse JC, Giedd JN, Rumsey JM, Vaituzis AC, Hamburger SD, Rapoport JL. Regional MRI measurements of the corpus callosum: A methodological and developmental study. Brain \& Development. 1996; 18(5):379-388. [PubMed: 8891233]

Rivera SM, Reiss AL, Eckert MA, Menon V. Developmental changes in mental arithmetic: Evidence for increased functional specialization in the left inferior parietal cortex. Cerebral Cortex. 2005; 15(11):1779-1790. [PubMed: 15716474]

Scarabino, T.; Di Salle, F.; Esposito, F.; Tosetti, M.; Armillotta, M.; Agati, R., et al. Chapter 7: 3.0 T Diffusion Studies. In: Salvolini, U.; Scarabino, T., editors. High Field Brain MRI. New York: Springer; 2006.

Schmithorst VJ, Holland SK, Ret J, Duggins A, Arjmand E, Greinwald J. Cortical reorganization in children with unilateral sensorineural hearing loss. NeuroReport. 2005; 16(5):463-467. [PubMed: 15770152]

Simon O, Mangin JF, Cohen L, Le Bihan D, Dehaene S. Topographical layout of hand, eye, calculation, and language-related areas in the human parietal lobe. Neuron. 2002; 33(3):475-487. [PubMed: 11832233]

Song SK, Sun SW, Ju WK, Lin SJ, Cross AH, Neufeld AH. Diffusion tensor imaging detects and differentiates axon and myelin degeneration in mouse optic nerve after retinal ischemia. NeuroImage. 2003; 20(3):1714-1722. [PubMed: 14642481]

Song SK, Yoshino J, Le TQ, Lin SJ, Sun SW, Cross AH, et al. Demyelination increases radial diffusivity in corpus callosum of mouse brain. NeuroImage. 2005; 26(1):132-140. [PubMed: 15862213]

Temple E, Posner MI. Brain mechanisms of quantity are similar in 5-year-old children and adults. Proc Natl Acad Sci U S A. 1998; 95(13):7836-7841. [PubMed: 9636237]

Toga AW, Thompson PM, Sowell ER. Mapping brain maturation. Trends Neurosci. 2006; 29(3):148159. [PubMed: 16472876]

van Eimeren L, Niogi SN, McCandliss BD, Holloway ID, Ansari D. White matter microstructures underlying mathematical abilities in children. NeuroReport. 2008; 19(11):1117-1121. [PubMed: 18596611]

van Eimeren L, Grabner RH, Koschutnig K, Reishofer G, Ebner F, Ansari D. Structure-function relationships underlying calculation: A combined diffusion tensor imaging and fMRI study. Neuroimage. 2010; 52, 1(1):358-363. [PubMed: 20382234] 
Wakana S, Caprihan A, Panzenboeck MM, Fallon JH, Perry M, Gollub RL, et al. Reproducibility of quantitative tractography methods applied to cerebral white matter. NeuroImage. $2007 ; 36(3): 630$ 644. [PubMed: 17481925]

Wakana S, Jiang H, Nagae-Poetscher LM, van Zijl PC, Mori S. Fiber tract-based atlas of human white matter anatomy. Radiology. 2004; 230(1):77-87. [PubMed: 14645885]

Wood G, Nuerk HC, Willmes K. Neural representations of two-digit numbers: A parametric fMRI study. NeuroImage. 2006; 29:358-367. [PubMed: 16253524] 


\section{Arabic Numerals}

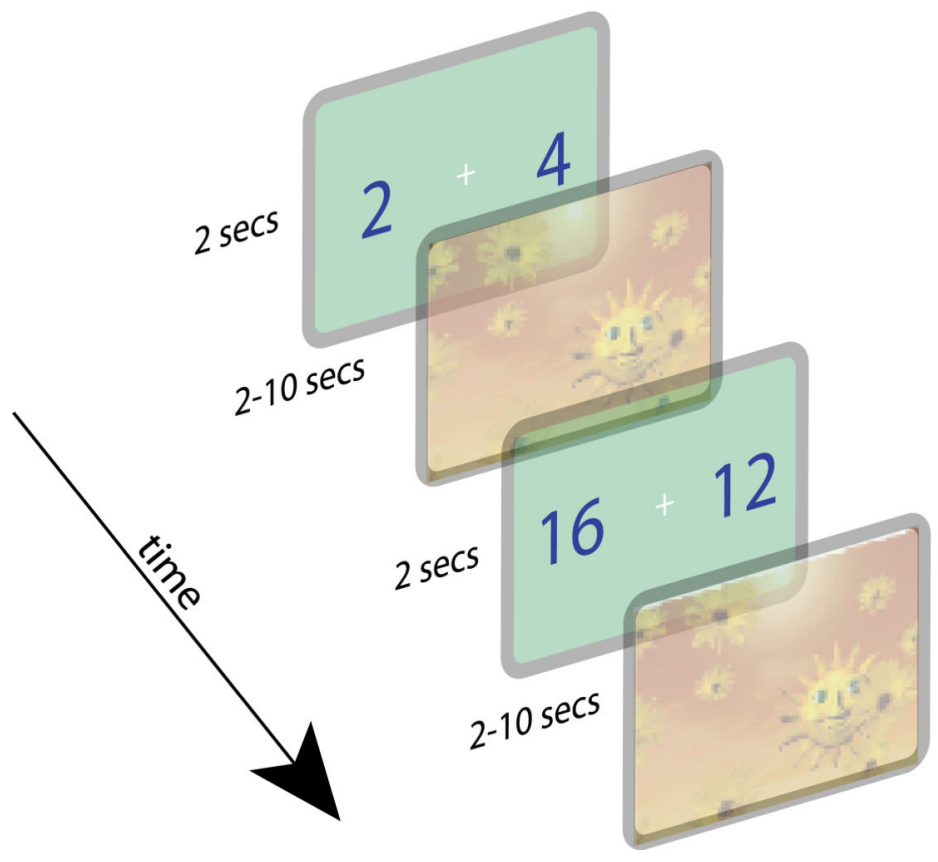

Dot Arrays

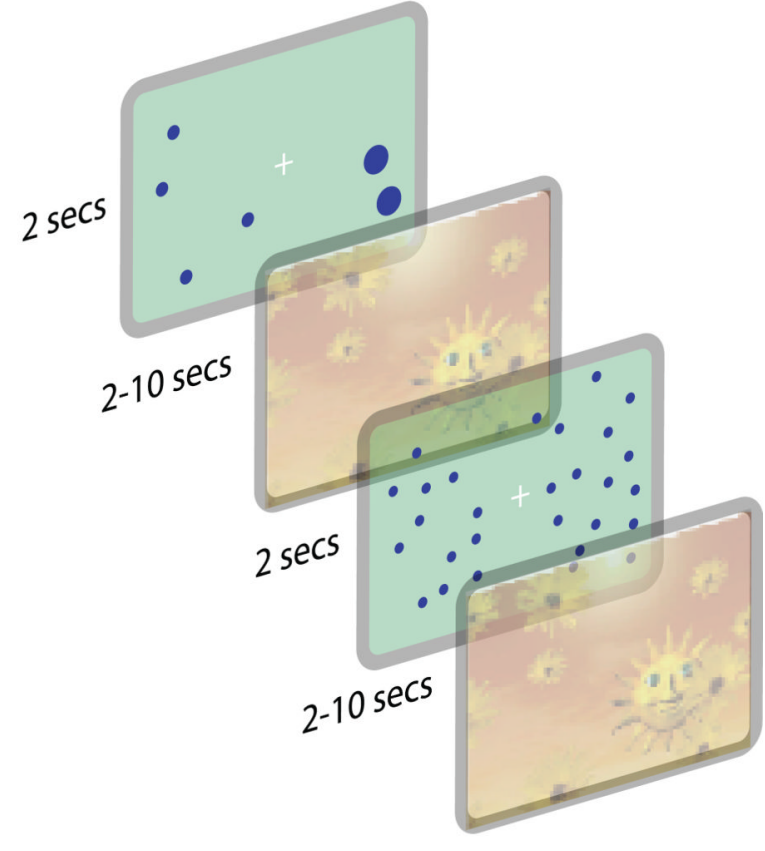

Figure 1.

The experimental task was to press a joystick button corresponding to the side of the screen with the larger numerical value. Subjects completed a Symbolic version of this task with Arabic numeral stimuli and a Non-symbolic version with arrays of dots as stimuli. Correct responses resulted in a reward screen: a picture of a bright sun and incorrect responses resulted in a black screen. Each trial was a maximum of two seconds after which the program would advance to the next trial. 


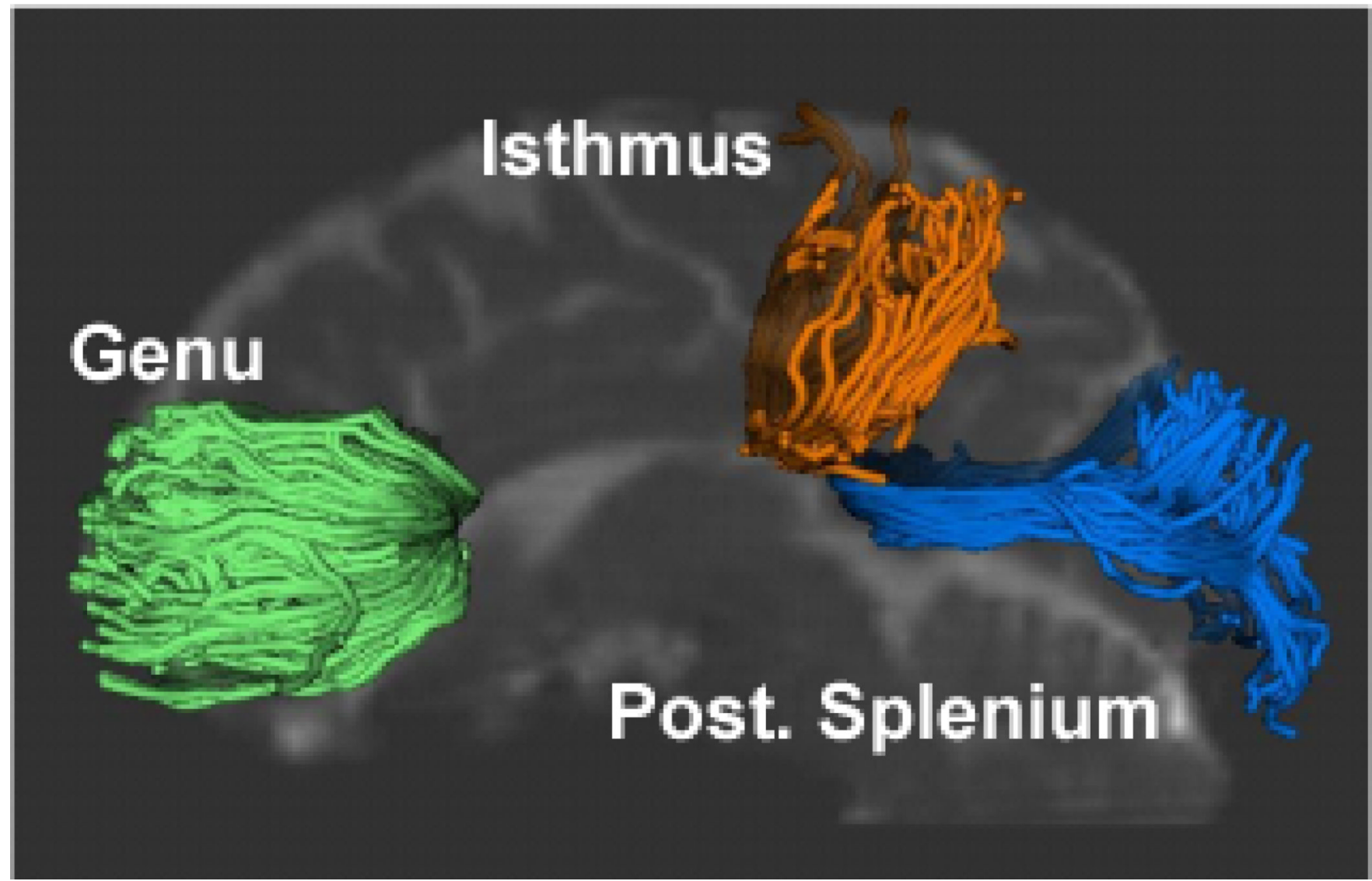

Figure 2.

The three tracts of the corpus callosum that were analyzed in the current study: fibers that crossed the genu (prefrontal cortex), the isthmus (superior parietal cortex), and the splenium (occipital cortex). Tracts are shown for a representative child subject. 


\section{Fractional Anisotropy}
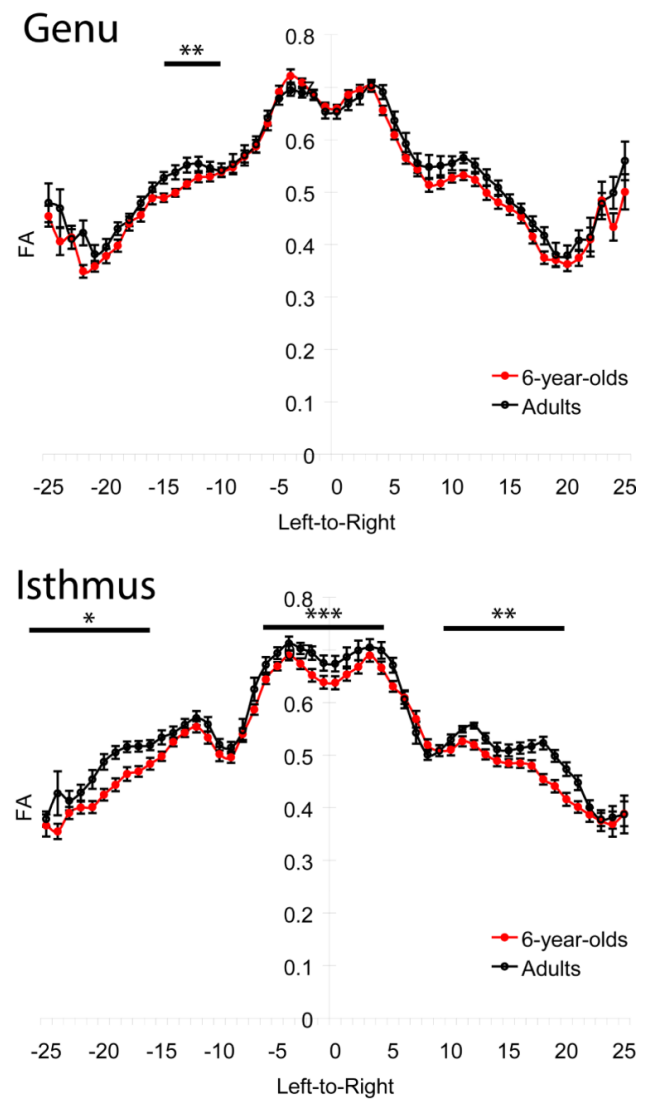

Splenium $\quad 0.9$

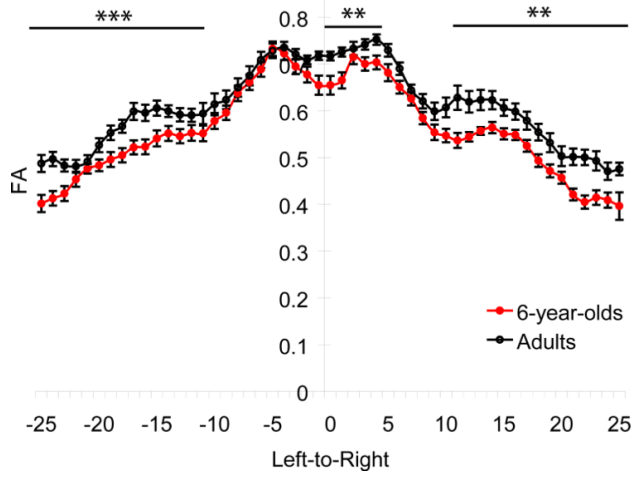

Figure 3.

Fractional anisotropy (FA) values for the fibers that traversed the genu (top), isthmus (middle), and splenium (bottom). The $\mathrm{x}$-axis is left-to-right position in millimeters and the $\mathrm{y}$ axis is the FA value. Children are shown in red and adults in black. Difference between children and adults are highlighted with black bars at the top of each panel $\left(* \mathrm{p}<.05,{ }^{*} \mathrm{p}<\right.$. $01, * * * \mathrm{p}<.001$; FDR corrected $\alpha=.05$ ). SEM is shown by error bars. 


\section{Axial Diffusivity}
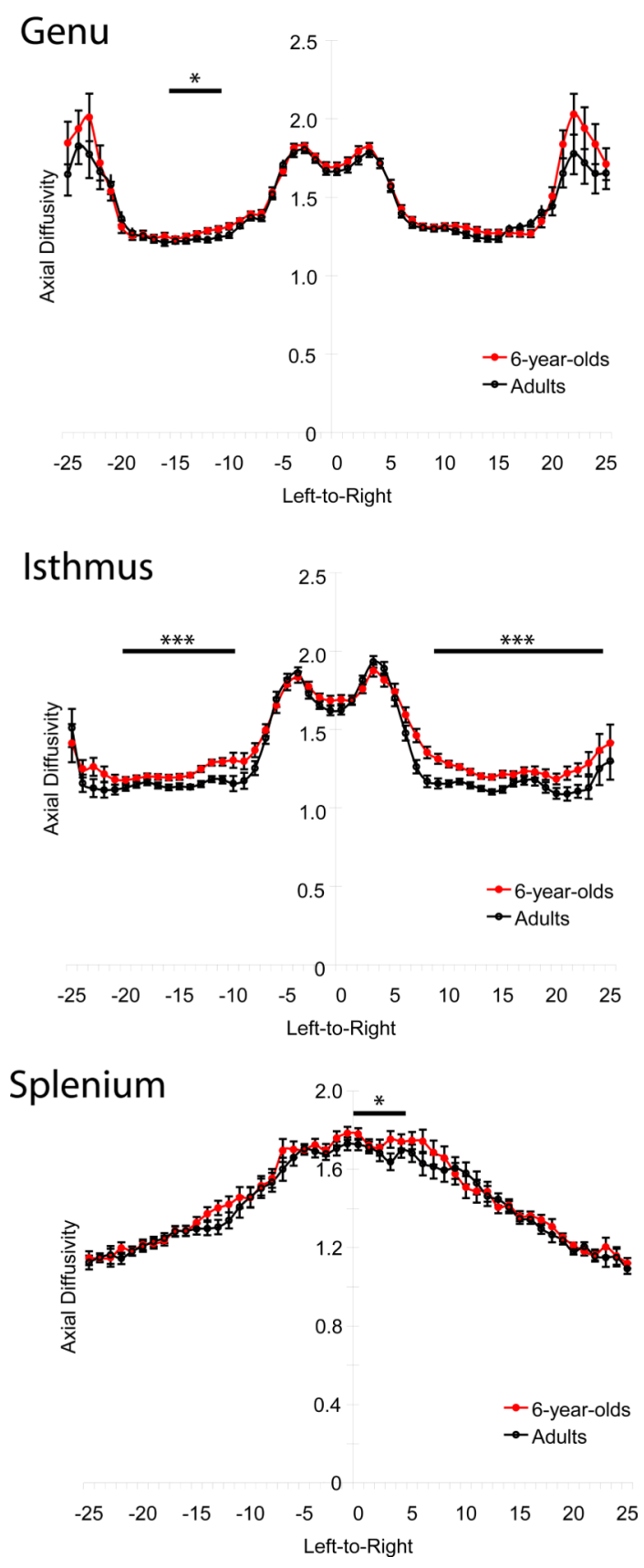

Figure 4.

Axial diffusivity (AD) values for fibers that crossed the genu (top), isthmus (middle), and splenium (bottom). The x-axis is left-to-right position in millimeters and the $y$-axis is the AD value presented in units of $10^{-3} \mathrm{~mm}^{2}$ per second. Children are shown in red and adults in black. Difference between children and adults are highlighted with black bars at the top of each panel $\left(* \mathrm{p}<.05, * * \mathrm{p}<.01,{ }^{* * *} \mathrm{p}<.001\right.$; FDR corrected $\left.\alpha=.05\right)$. SEM is shown by error bars. 


\section{Radial Diffusivity}
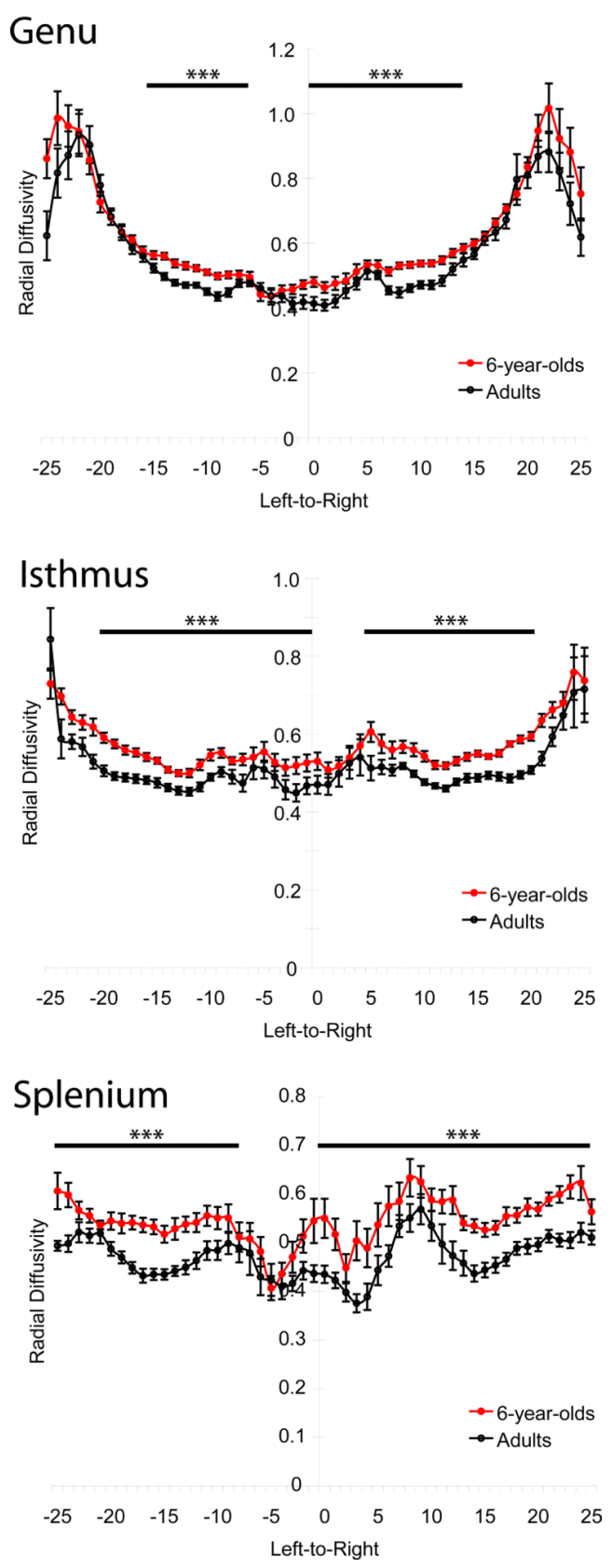

Figure 5.

Radial diffusivity (RD) values for the fibers traversing the genu (top), isthmus (middle), and splenium (bottom). The $\mathrm{x}$-axis is left-to-right position in millimeters and the $\mathrm{y}$-axis is the RD value presented in units of $10^{-3} \mathrm{~mm}^{2}$ per second. Children are shown in red and adults in black. Difference between children and adults are highlighted with black bars at the top of each panel $\left(* \mathrm{p}<.05, * * \mathrm{p}<.01,{ }^{* * *} \mathrm{p}<.001\right.$; FDR corrected $\left.\alpha=.05\right)$. SEM is shown by error bars. 

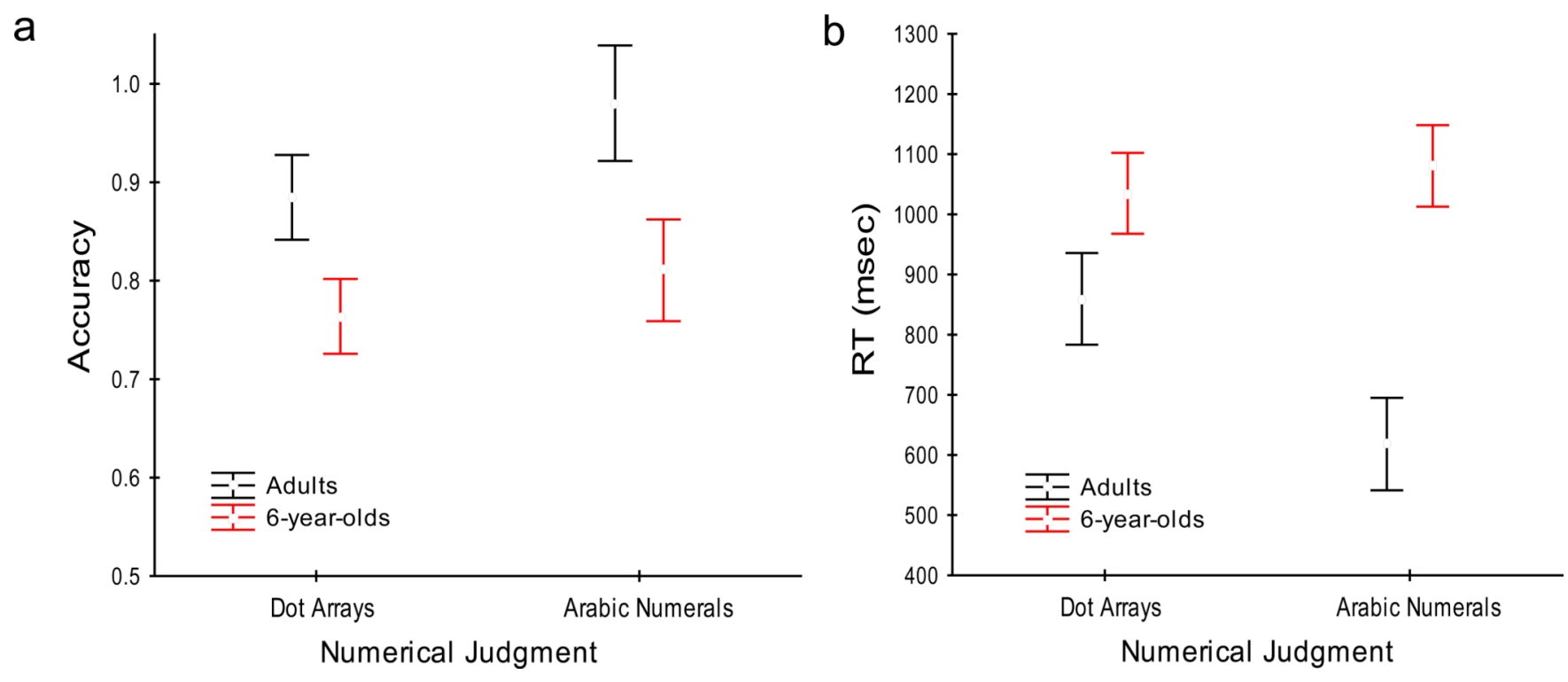

Figure 6.

Behavioral performance on the two numerical tasks. Accuracy (a) and RT (b) are shown for adults (black) and children (red) with $95 \%$ confidence intervals denoted by the vertical bars. 
a

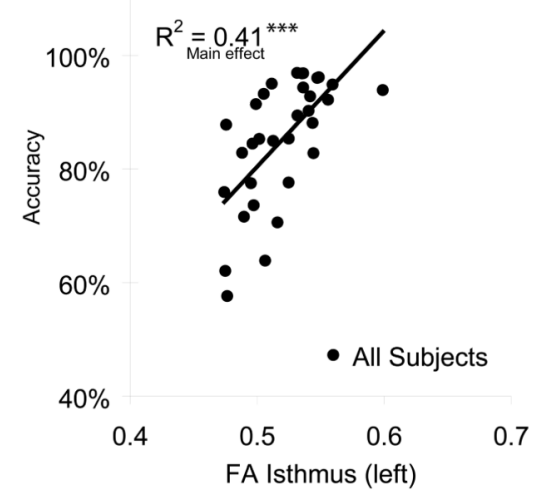

C

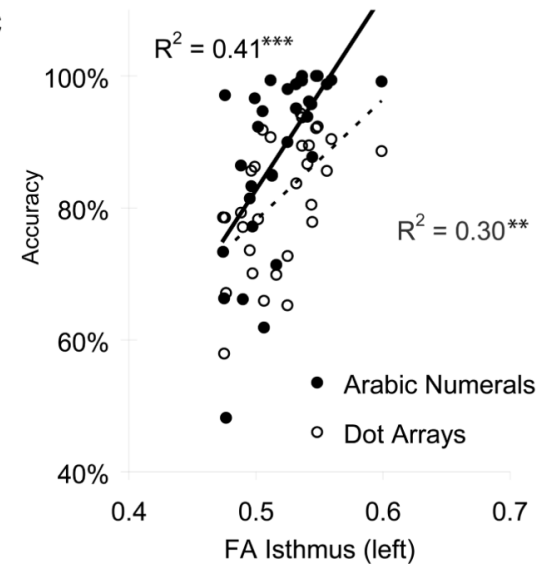

e

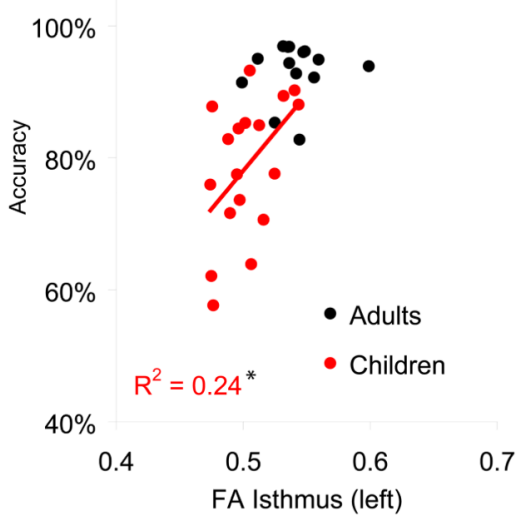

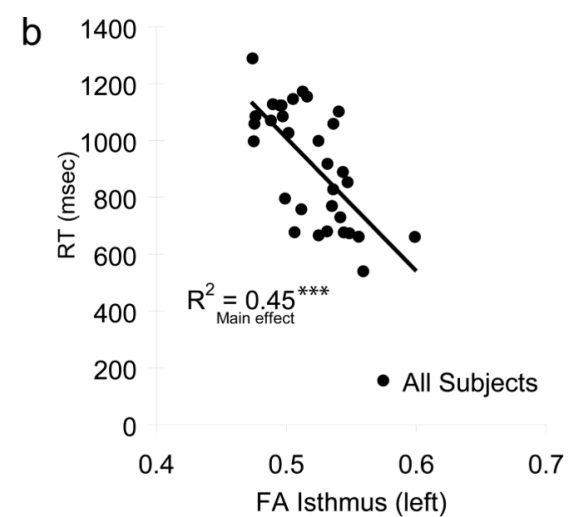

d

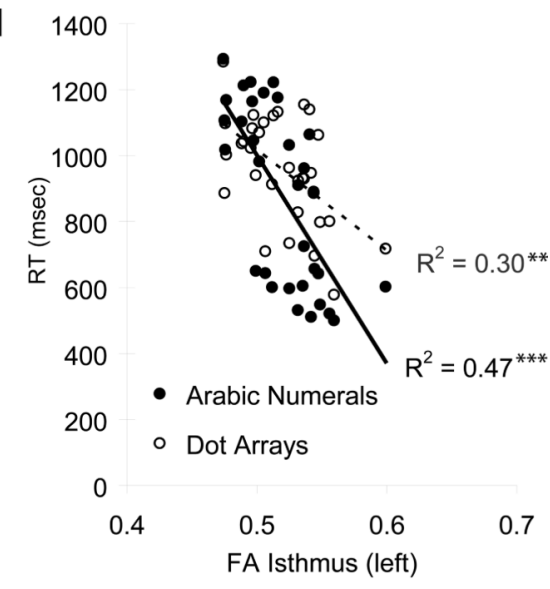

f

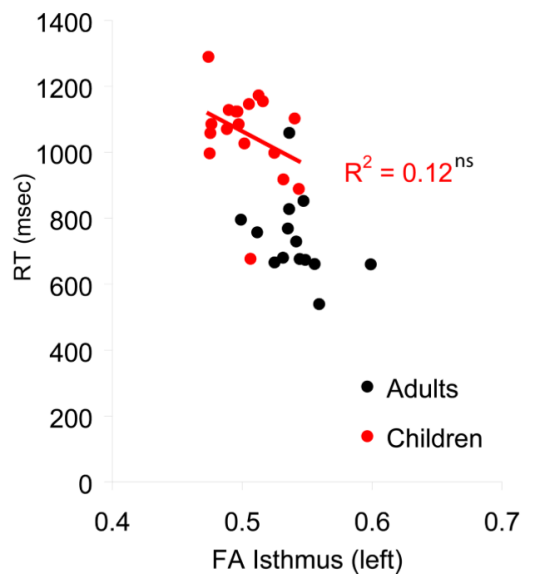

Figure 7.

Brain-behavior correlations. There was an overall correlation between accuracy (a), RT (b), and FA of the fibers crossing the left isthmus. The overall correlation was significant for each of the two numerical judgment types (Arabic numeral and Dot Arrays) in accuracy (a) and RT (b). The overall correlations for children only (red) are shown in panels e and f for accuracy and RT, respectively. Only the correlation between FA of the left isthmus tract and accuracy was significant within the child-only sample $(* \mathrm{p}<.05, * * \mathrm{p}<.01, * * * \mathrm{p}<.001)$. 